\title{
UMLS Concept Unique Identifier
}

National Cancer Institute

\section{Source}

National Cancer Institute. UMLS Concept Unique Identifier. NCI Thesaurus. Code C42718.

In the Unified Medical Language System (UMLS) of the National Library of Medicine (NLM) the concept unique identifier (CUI) is an 8-character identifier beginning with the letter "C" and followed by 7 digits. Each concept is assigned such a CUI. The CUI has no intrinsic meaning but remains constant through time and across versions. 